\title{
The Secret of the Titius-Bode Law: A New Theory on How Our Planetary System Came Into Existence
}

\author{
Hans Merk $1^{1}$ \\ ${ }^{1}$ Am Kirchsteig 4 - 92637 Weiden, Germany \\ Correspondence: Hans Merkl, Am Kirchsteig 4 - 92637 Weiden, Germany. E-mail: flacherhans@gmx.de
}

Received: November 26, 2019

Accepted: December 31, 2019 Online Published: January 31, 2020

doi:10.5539/jgg.v11n4p58

URL: http://dx.doi.org/10.5539/jgg.v11n4p58

\begin{abstract}
Our planetary system still has several unsolved riddles. One of them is the Titius-Bode law. With the aid of this law, it is easy to find the distances of planets from the sun. For many astronomers, this is coincidence. They argue that there is no known physical mechanism that generates a particular sequence of planets' distances. However, if one investigates the structure of the law, it quickly becomes clear that the Titius-Bode law is directly connected with the formation of planets. Our planets did not come into existence through so-called accretion. At the beginning of its existence, the sun was presumably a T-Tauri star. These are young stars in the process of their formation. They pulsate irregularly, thereby accelerating clouds of plasma in the surrounding dust disk. Each of these eruptions thus generated a planet. This of course goes much more quickly than if they had to be formed from the dust of planetary disks. This new theory not only describes how the planets and the distances of the planets came into existence. It also gives a new description of how the moon came into existence, the cause for large moon craters, the slow rotation of Venus, the formation of the asteroid belt, the high iron content of the planet Mercury, and the sun's loss of rotational impulse, among other things.
\end{abstract}

Keywords: planets, sun, T-Tauri stars, plasma, magnetic phenomena

\section{Introduction}

Every astronomer knows the Titius-Bode law. It was already mentioned in 1766 by the German astronomer Johann Daniel Titius. It is a simple rule to determine the planets' distance from the sun. Because there is no certain explanation for this rule, the majority of astronomers assume that this law is a coincidence. But the astronomers Tim Bovaird and Charles H. Lineweaver of the Australian National University in Canberra analysed 31 extrasolar planetary systems and noticed that they follow the Titius-Bode formula at least more precisely than the celestial bodies in our planetary system.

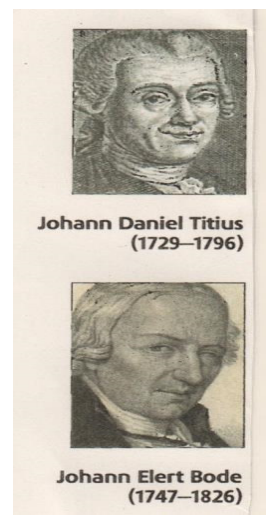

Of the 31 systems investigated, the planets in 26 systems lined up according to the Titius-Bode law but more precisely than in the solar system. Three systems roughly correspond to the solar system. Two systems do not fit the scheme.

It is thus unlikely that the Titius-Bode law is based on a coincidence.

Mathematically, the law is described by the following formula: 


$$
\mathrm{E}=0.4+0.3 \times 2^{\mathrm{n}}
$$

$\mathrm{E}=$ distance in astronomical units

Exponent $\mathrm{n}$ has the values $-\infty, 0,1,2, \ldots$

This formula, however, is not very clear. Therefore, the next chapter provides a more detailed account.

\section{The law}

Each of the following numbers stands for one planet

$\begin{array}{ccccccccc}0 & 3 & 6 & 12 & 24 & 48 & 96 & 192 & 384 \\ \text { Mercury } & \text { Venus } & \text { Earth } & \text { Mars } & \text { Asteroids } & \text { Jupiter } & \text { Saturn } & \text { Uranus } & \text { Neptune }\end{array}$

As can be seen, each number after the 3 is twice that of the previous value. Now add the number 4 to each number from this row. If the resulting value is then divided by 10, one receives the distance of the planets from the sun in astronomical units (abbreviated: $\mathrm{AU} \bumpeq$ = major semi-axis of the earth - sun). That is: $1 \mathrm{AU} \triangleq 150$ million kilometres.

The distance of planets calculated in this way in $\mathrm{AU}$ is:
0.4
0.7
1.0
1.6
2.8
5.2
10
19.6
38.8

The actual distance (according to Astronomie in Theorie u. Praxis [Astronomy in Theory and Practice], E. Wischnewski)
0.39
0.72
1.0
1.52
2.9
5.2
9.55
19.2
30.1!

As the numbers in the top row show, the value of the number doubles from planet to planet. This cannot be a coincidence. On the contrary, this is a function of the natural logarithm. This is found in many natural processes. But number 24 is not a planet. This is the location of the asteroid belt between Mars and Jupiter. Why is there no planet orbiting there? There are two different theories explaining this. One states that a planet did orbit there at one time. But after a collision with another celestial body, it broke into pieces. A part of the shower of rubble may have been the cause of the "heavy bombardment" 3.9 billion years ago, the signs of which are still visible on the moon. Others think that the strong gravitational influence of Jupiter prevented the formation of a planet. But with the last planet, Neptune, the actual distance is no longer correct! It is obviously an outlier in the row. At a distance of 39.5 AU, i.e., only 0.5 AU from the theoretical distance of Neptune, the small planet Pluto is oddly located.

\section{But how can one explain how these planetary distances came into existence according to the pattern shown above?}

The simplest explanation is that the sun pulsated during its formation and flung out large quantities of matter with each of these explosions into the protoplanetary gas and dust disks. This hot discharged matter in the state of plasma generates a magnetic field (moving charge) through the fast motion, out of which planets are formed.

The formation of a planetary system through a pulsating star can in fact be observed in our Milky Way with T-Tauri stars (Image below). T-Tauri stars can reach a size of up to three times the mass of the sun. These are young stars that are less than one million years old. They are surrounded by a circumstellar dust and gas disc and are in a phase where they still contract and are not yet in static equilibrium. Therefore, they tend to more or less intense, irregular explosions.

Our sun presumably also went through this stage of a T Tauri star.

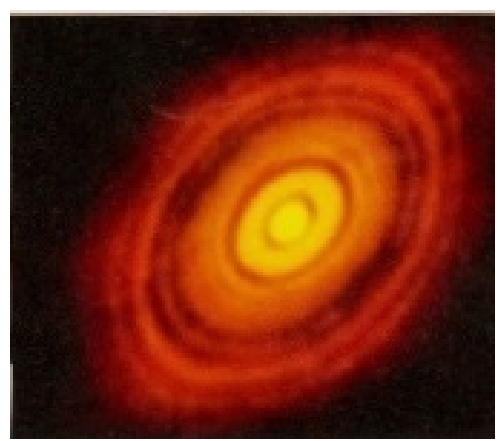

Figure 1. Protoplanetary disk around the star HL Tauri

New telescopes allow astronomers to observe the formation of planets with other stars.

This also permits drawing conclusions as to the birth of our solar system. 


\section{Our planets - children of the sun}

Every shockwave from an explosion in the centre, the location of the forming sun, extended outward in the disk at this stage of formation. This is shown in the image above. Discharged plasma becomes compressed during this process. Concentric circles are formed pushing toward the matter to the edge of the protoplanetary disk.

In this context, it is therefore worth investigating more closely plasma and its interactions with normal matter since this is important not only for the formation of stars, but also for the formation of planets. While plasma is nothing other than ionized gas, the interactions in plasma and gases differ greatly. Therefore, researchers consider plasma to be a fourth state of matter, alongside solid, liquid, and gaseous forms. Plasma is everywhere in the cosmos. The sun is an enormous plasma ball. In the cosmos, plasma is completely normal. - Unlike neutral gases, where atomic and molecular interactions predominate, particles of plasma interact with microparticles through their electrical forces and form so-called "complex plasma." This happens, for example, in the cosmos if normal plasma mixes with dust. Although the dust particles measure only $1 / 10,000^{\text {th }}$ of a millimetre, they are several billion times heavier than atoms, but they have an electrical charge because of the ions (Gregor Morfill, Hubertus Thomas).

Because the matter at the edge of the protoplanetary disk rotated more slowly than in the centre of the disc, the material that was pressed outward from the explosions was slowed down and thus swirled. This swirled "complex plasma" (moving charge) generated strong magnetic forces which compressed the plasma-like matter until gravitation formed from it a planetary embryo. As it orbited in the protoplanetary disk, it assembled further matter. It collected the surrounding dust masses during this process thereby growing relatively quickly into the final planet. This also explains why only one body exists in a planetary orbit, namely the one large vortex where the majority of the matter could bind together. The remaining dust and gaseous residue was driven out of the disk by solar wind. Therefore, the planets were formed from the outside to the inside of the planetary disk. However, it may actually be possible that gas planets were formed much closer to the forming sun but they drifted outward because of their orbital speed until they were slowed down by the slower gas in the outer disk and reached a stable orbit.

This theory of course challenges the standard theory of planet formation. As with star formation, there are also various theories about the formation of planets. The currently recognized theory assumes that the dust particles of the protoplanetary disk merged into ever larger particles. If the bodies were large enough, gravity ensured their further growth such that ultimately minute planets, so-called planetesimals, were created. These then grew into today's large planets through additional clashes. This is the so-called accretion theory. - However, I believe, there are two objections to it:

Comprehensive experiments conducted at the University of Braunschweig/Germany in which zero gravity was generated by field trials to simulate the conditions in the cosmos, were not particularly successful. While these dust particles adhered together through electrostatic forces, at a certain size, they bounced off each other when they collided. Therefore, the process of planet formation through colliding dust particles and subsequent minute planet formation takes too long.

From the time when the sun starts to glow due to nuclear fusion, all the dust in the disk is blown out of the disk to the edge by solar wind. Planet formation through dust in the disk must have already been completed by this time. Additionally, the question is raised how, in this process, did all the planets get their rotational impulse in the same direction? If bodies collide into each other from all possible directions, the rotational impulses emerging from this would in fact have to be in different directions.

Of course, according to this standard theory, the dust particles will be able to connect to larger bodies and populate the solar system, but the large planets presumably came into existence through swirls.

\section{Planet Distances Emerge from an Irregularly Pulsating Sun}

By means of the material made of gas and dust falling on a shared centre, a rotating, protoplanetary disk formed because of the pirouette effect. In the centre of the spherical material accumulation, the location of the future sun, nuclear fusion started when pressure and temperature were high enough. But it is not to be assumed that the fusion in the centre encompassed ever larger surrounding areas, little by little. The fusion zone grew slowly and the sun shined. On the contrary, the first ignition of this hydrogen bomb in the centre again separated the masses of gas and dust, which were pressed by gravitation to the middle, like an explosion. But the fusion thereby came to an end because the pressure and temperature on the inside were too low. But gravitation still continued to pull the gas and dust towards the inside. The more the material pressed to the centre, the quicker the protoplanetary disk spun. Because of this, the outer disk edge drifted toward the inside. The intervals between explosions also shortened because the mass and pressure in the centre, which are necessary for another fusion, were attained faster and faster. Like many processes in nature, this shortness of time of the consequences of explosions took place in accordance 
with a function of the natural logarithm. This is reflected in the Titius-Bode law and thus clearly explains why this law describes the distances of planets. Every eruption hurled large quantities of material into the planetary gas and dust disks. Quickly rotating planets could develop in a relatively short time from the swirls of these masses of gas and dust, which were in the state of a "complex plasma," first by magnetic forces and then by gravitation. The planets thus were formed out of the explosion residue from the sun in labour. This interaction continued until the pressure from the pushed masses of gas at the centre was as high as the internal explosion pressure. The forces were then in equilibrium and our sun was born.

\section{Discussion}

\subsection{At the End of Development}

During this phase, when the young sun began to shine, the strong solar wind blew dust and gas away from the planetary disk. At this time, the planets should have therefore already existed, since at that time, the solar wind dissolved the disc. Astronomers observed that young sun-like stars in our cosmic neighbourhood have a stellar wind that is up to one-thousand times greater than our sun has at this time. Thus, no matter is left over for planet formation. Observations of extrasolar systems have shown that this lasts only 2 to 3 years. At that time, the new star and its planets are visible. No material is left over for further planet formation. That would also explain why always only one planet exists in a planetary orbit. It is namely the one, the vortex of which drew all the material to itself. The solar wind blew the remaining matter in the disk to the edge of the planetary system in the region beyond Pluto in the Kuiper belt. The icy, small planets and comets formed out of this residual matter. Further still outside at the edge of the solar system, in the region of the Oort cloud, matter from comets originating from there show that the residual material of the protoplanetary disk had already mixed with star dust there. But it is not to be assumed that this principle of planet formation always applies. With an extrasolar planetary system, enormous gas planets were discovered that go around their mother star in a very close orbit of only a few days. - It is conceivable that the rotational speed of the emerging star became so strong because of the great material influx from the disc that the star lost a part of its external mass due to the high centripetal inertia and supported by a star eruption. From this, a large gas planet formed, which goes around the star closely. The mass of the planet is not relevant for the orbit speed. What is critical is only the distance from the star and the star's mass. Therefore, how a planetary system is being formed obviously depends on the overall available quantity of the matter involved.

\section{The Formation of the Moon}

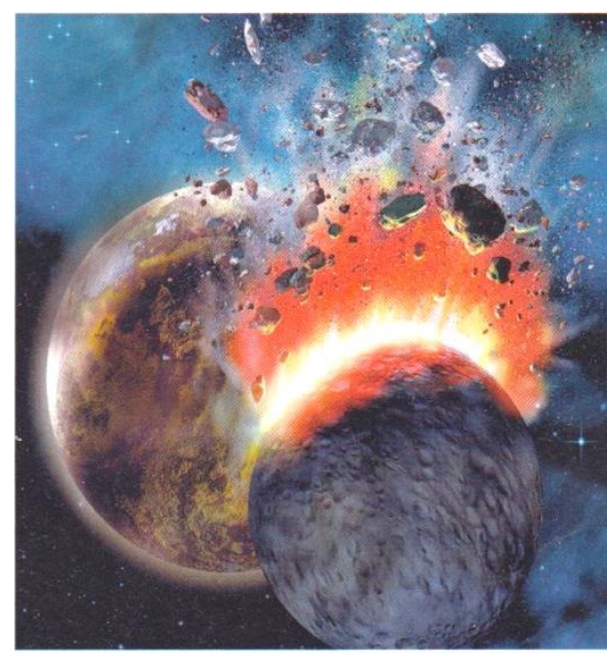

Figure 2. The formation of the moon from a collision with the hypothesized planet Theia presumably did not happen this way

The vortex theory explaining the formation of planets allows for the inference that the earth-moon system did not come into existence by a collision of the primordial earth with the hypothesized planet Theia. This theory developed by the astronomer Hartmann (USA) is currently still very popular. It is supported above all by the rock samples brought back from the moon by astronauts. However, the most recent research results published in the journal Nature Geoscience by researchers working with Junjun Zhang have come to the conclusion that the moon is the earth's geochemical twin. They investigated the frequencies of two isotopes of the metal titanium in the 
moon rock samples. This showed that the relationship of titanium 50 to titanium 47 in the moon rock did not differ from the distribution in the earth's crust by more than $0.0004 \%$. This is surprising because the isotope frequencies otherwise vary in the solar system up to a hundred times more strongly. Even for oxygen, silicon, chromium, and tungsten, the isotope relationships are similar, as Mathias Meier from the Swedish University of Lund emphasizes in a supporting article in Nature Geoscience.

These results challenge the current model of moon formation. If there were a collision with Theia, the material of the moon would differ from that of the earth. Because of these results, the earth and the moon can definitely be considered twins. They presumably came into existence from a double vortex. - At the beginning, in the phase when both celestial bodies, earth and moon were still very close to each other, the rushing tidal bulge from the earth's molten rock masses accelerated the moon. The moon was able to distance itself from the earth because of this acceleration. However, this also led to a deceleration of the earth's rotation. In nature, there is nothing without a reason. After the earth's surface had cooled off, fortunately a large quantity of water formed very quickly on the earth. In its early days, the earth was actually a water planet. The causes of the earth's early abundance of water were detailed by Merkl. The moon thus continued to distance itself from the earth because the gravitation between the earth and moon continued to generate a rushing tidal bulge in the early oceans. Such a tidal bulge accelerated the moon more. In this way, the moon continued to distance itself from the earth. Even today this effect still causes an acceleration of our moon. Because of this, the moon still becomes more distant from the earth by around $4 \mathrm{~cm}$ annually.

The huge impact basins on the front side of the moon are conspicuous. During the time of the heavy bombardment 4.1 to 3.8 billion years ago, huge meteorites struck there. The craters filled up with magma from the inside of the moon and thus formed the "mare" that are still visible today. But why did these large impacts only come into existence on the front side? Interestingly, now an answer can be given to this from a method of accelerating spacecraft that is frequently applied in space technology. By having spacecraft fly closely to planets, they are accelerated by gravity and continue flying at a higher speed. The same effect sped up asteroids and comets that flew by the earth during the heavy bombardment around 3.9 billion years ago. If the moon happened to be in the "line of fire" with its orbit around the earth, these accelerated celestial bodies struck the front side of the moon at a high speed since this side of the moon always turns toward the earth because of synchronous rotation. These huge craters formed due to the high speed generated by the "swing by." This effect is absent on the back side of the moon. Only one large impact crater is located here.

\section{Conclusions}

According to this theory of the formation of our planetary system by means of a pulsating sun embryo, the outer planets, i.e., the gas giants, starting with Neptune, Uranus, Saturn, and Jupiter, initially formed from the outside to the inside in temporal succession. It is to be assumed that at this time the protoplanetary disk was still being formed and thus was surrounded by large volumes of gas. Because of this, these gas giants were able to become extremely large. Then, at certain intervals, based on eruptions from the sun, the asteroid belt followed, as did the planets Mars, Earth, Venus, and Mercury.

After the sun shined, the strong solar wind blew the residual material from the inner protoplanetary disk toward the outside, such that the gas planets were able to accumulate additional material during this phase.

The asteroid belt at the boundary between the large gas planets and the small rock planets is a special case, the formation of which has not yet been explained. But it could be that, after the formation of Jupiter, the "complex plasma" discharged again from the sun in the region of today's asteroid belt cooled down so much that it turned back into dust and neutral gas. Thus, the impulse from this solar material emitted again could no longer produce any magnetic field to form a planet. The dust changed from rock formations and small planets into this chaotic asteroid belt due to electrostatic and gravitational forces, i.e., due to so-called accretion. But this process would have to take place in extrasolar planetary systems also and be discovered there.

A further secret is Venus with its very slow rotation. In addition, it rotates in opposite direction as opposed to the other planets of our solar system. Some astronomers assume that a large celestial body hit Venus and that created this anomaly. - However, because planets presumably formed from a vortex of matter, it definitely could be that Venus formed from a double vortex. However, unlike the moon-earth system, the two roughly similarly sized vortices crashed into each other and thus annihilated its original rotational impulse.

With its large iron core, Mercury also gives reason for speculations. Because of its nearness to the sun, the strong solar wind might have simply blown away the lightweight components of the material vortex, out of which Mercury formed. - Or might it be that the young sun rotated at the end of its formation so quickly that it, like an 
enormous centrifuge, transported the heavy elements to the edge of the solar disk, which formed the inner rock planets due to the final explosions? This might explain why the planet Mercury has such a large iron core.

Because of these explosions that created our planets, the sun of course lost a part of its rotational impulse every time.

Our earth, with a large moon, a lot of water, and the suitable distance to the sun, is actually a unique stroke of luck explaining how life came into being. But here it is to be assumed that also in extrasolar systems, the formation of planets by vortices, which affect the gas and dust masses surrounding the star, earth-like planets, perhaps even with a large moon, should be found more frequently in our galaxy.

\section{References}

Bovaird, T., \& Lineweaver, C. H. (2013). Exoplanet predictions based on the generalized Titius-Bode relation. Monthly Notices of the Royal Astronomical Society, 435(2), 1126-1138. https://doi.org/10.1093/mnras/stt1357

Merk1, H. (2015). On the Origin of Water on Earth and Mars-A New Hypothesis. Journal of Geography and Geology, 7(2), 1-5. https://doi.org/10.5539/jgg.v7n2p1

Merkl, H. (2017). Wasser der Erde-ein Produkt der Sonne? Eine Hypothese über die Herkunft des Wassers auf der Erde. Zeitschrift der Deutschen Gesellschaft für Geowissenschaften, 168(2), 211-216. https://doi.org/10.1127/zdgg/2017/0108

Morfill, G., \& Hubertus, T. (1993). Plasma - eine komplexe Materie [Plasma - A complex matter]. Max-Planck-Institut, Garching/Muenchen, Germany.

Zhang, J., Dauphas, N., Davis, A. M., Leya, I., \& Fedkin, A. (2012). The proto-Earth as a significant source of lunar material. Nature Geoscience, 5(4), 251-255. https://doi.org/10.1038/ngeo1429

\section{Copyrights}

Copyright for this article is retained by the author(s), with first publication rights granted to the journal.

This is an open-access article distributed under the terms and conditions of the Creative Commons Attribution license (http://creativecommons.org/licenses/by/4.0/). 